\title{
Effects of Low Intensity Pulsed Ultrasound on Expression of Bax and Bcl-2 in Premature Ovarian Failure Mice Induced by 4-Vinylcyclohexene Diepoxide
}

\section{Haopeng Xu}

Chongqing Medical University

Yi Xia

Chongqing Medical Unversity

Juan Qin

Guiyang Maternal and Child Health Hospital

Jie Xu

Chongqing Medical Unversity

Chongyan Li

Chongqing Medical Unversity

\section{Yan Wang ( $\square$ wangyancq@cqmu.edu.cn )}

Skate Key Laboratory of Ultrasound in Medicine and Engineering;College of Biomedical

Engineering;Chongqing Key Laboratory of Biomedical Engineering;Chongqing Medical University https://orcid.org/0000-0001-6555-9946

\section{Research}

Keywords: POF, LIPUS, VCD, apoptosis, Bax, Bcl-2

Posted Date: January 18th, 2021

DOI: https://doi.org/10.21203/rs.3.rs-146916/v1

License: (c) (i) This work is licensed under a Creative Commons Attribution 4.0 International License.

Read Full License

Version of Record: A version of this preprint was published at Reproductive Biology and Endocrinology on July 20th, 2021. See the published version at https://doi.org/10.1186/s12958-021-00799-w. 


\section{Abstract}

\section{Background}

Premature ovarian failure (POF) is a common disease in the field of gynecological. This study was performed to determine the effects of low intensity pulsed ultrasound (LIPUS) on granulosa cells (GCs) apoptosis and protein expression of Bax and Bcl-2 in 4-vinylcyclohexene diepoxide (VCD)-induced POF mice. The aim of this research is to investigate the mechanisms of LIPUS on ovarian function and reserve capacity.

\section{Methods}

The current POF mice model was administrated with VCD $(160 \mathrm{mg} / \mathrm{kg})$ by intraperitoneal injection for 15 consecutive days. The mice were divided into POF group, LIPUS group and control group. In the LIPUS group, the right ovary of mice was treated by LIPUS (acoustic intensity was $200 \mathrm{~mW} / \mathrm{cm}^{2}$, frequency was $0.3 \mathrm{MHz}$, and duty cycle was 20\%) for 20 minutes, 15 consecutive days from day 16 . The mice of the POF group and control group were treated without ultrasonic output. The basic observation, Hematoxylin and eosin staining (H\&E staining), TUNEL assay and immunohistochemistry were applied to detect the results.

Results

The results showed that VCD can induce estrus cycle disorder, follicular atresia and GCs apoptosis in mice to establish POF model successfully. LIPUS significantly promoted follicular development, inhibited excessive follicular atresia and GCs apoptosis. The mechanism may be achieved by increasing the protein expression of $\mathrm{Bcl}-2$ and decreasing the expression of Bax in ovaries.

\section{Conclusions}

These findings have the potential to provide novel methodological foundation for the future research, which help treat POF patients in the clinic.

\section{Introduction}

Premature ovarian failure (POF), a common disease in the field of gynecological, is endocrinology pathologically characterized by amenorrhea, infertility, estrogen deficiency, and increased gonadotropin levels in women ${ }^{[1]-[2]}$. The incidence of POF is $1 \%$ before the age of 40 , which induces multiple health risks, including night sweats, insomnia, mood changes, blood pressure fluctuations, a series of syndromes of osteoporosis and cardiovascular disease ${ }^{[3]-[4]}$. Therefore, modeling and treatment of POF have been paid more attention in recent years.

According to different pathological manifestations, there are many approaches to model POF. The most common is irreversible pathogenesis of POF caused by chemotherapy drugs such as cyclophosphamide 
and busulfan, which could induce follicle loss, vascular damage and tissue fibrosis ${ }^{[5]-[7]}$. But the chemotherapy drugs damages other tissues and organs in addition to ovarian tissue and increase the risk of death during modeling ${ }^{[8]}$. Besides, iatrogenic surgery and gene knockout were also successful in establishing models of POF. However, these two methods have the disadvantage of simulating only part of POF and high costs respectively ${ }^{[9]-[10]}$. Our present study was performed on the POF mice model induced by 4-vinylcyclohexene diepoxide (VCD). VCD is a novel ovarian toxic substance that induces POF animal models to exhibit unique advantages in dynamic simulation of human ovarian decline ${ }^{[11]-[12]}$. Apoptosis of oocyte and granulosa cells (GCs) has been identified as one possible underlying mechanism for VCD-induced POF, which leads primordial and primary follicles to be destroyed ${ }^{[13]-[14]}$. Bcell lymphoma-2 (Bcl-2) and BCL2-Associated $\mathrm{X}(\mathrm{Bax})$ are regulators of primordial follicle initiation and apoptosis, and the ratio of $\mathrm{Bcl}-2$ /Bax determines whether cell apoptosis occurs when stimulated by apoptosis signal ${ }^{[15]-[16]}$.

At the same time, there are also many ways to treat POF, including hormone replacement therapy (HRT), ovarian transplantation, low temperature oocyte preservation technology and stem cell therapy etc ${ }^{[17]-}$ [21]. HRT is considered as the most commonly used treatment at home and abroad, but it is easy to increase the probability of endometrial cancer and breast cancer ${ }^{[17]}$. Low intensity pulsed ultrasound (LIPUS) is defined as a safe and effective therapy that has been studied in recent years and used to help treat many diseases ${ }^{[22]}$. As an emerging physical therapy, LIPUS can promote bone healing, soft-tissue regeneration, inhibition of inflammation and other applications ${ }^{[23]}$. It has also been found that LIPUS can help promote the recovery of ovarian histological structure in rats with ovarian injury induced by cyclophosphamide ${ }^{[24]}$.

Starting from the modeling and treatment of POF, we performed the effects of LIPUS in POF mice induced by VCD in this study. The aim of our study is to investigate ovarian function and reserve capacity, GCs apoptosis and related $\mathrm{Bcl}-2$ and $\mathrm{Bax}$. The results might introduce a new method to help treat POF in clinics in the future.

\section{Materials And Methods}

\section{Animal}

A total number of 12 female C57BL/ 6 mice (weight $20 \pm 2 \mathrm{~g}$ and age 6-8 weeks) used in this study were obtained from the Experimental Animal Center of Chongqing Medical University (production license number: SCXK 2018-0003). All experimental animals were maintained under standard conditions (humidity $45 \% \pm 5 \%$, temperature $23 \pm 5{ }^{\circ} \mathrm{C}$, light time $12 \mathrm{~h} / \mathrm{d}$ ). The animal experimental protocol was according to the ethical standards of the Experimental Animal Ethics Committee of Chongqing Medical University. Animal handling and experimental procedures were performed in accordance with the Guide for the Care and Use of Laboratory Animals issued by the Ministry of Science and Technology, China. All 
animal experiments were conducted at the Experimental Animal Center, Chongqing Medical University, China.

The mental state of the mice, diarrhea, hair color and activity were observed and recorded during the experiment.

\section{POF model establishment and grouping}

The mice were allowed to acclimatize for 1 week. Then, they were randomly divided into the three following groups with 4 mice per group: LIPUS group $(n=4)$, POF group $(n=4)$ and control group $(n=4)$. The VCD-induced POF mice model was established according to the literature ${ }^{[25]}$. The mice in the POF group and LIPUS group were intraperitoneally injected daily with VCD (Shanghai Aladdin Biochemical Technology Co. LTD, Shanghai, China; $160 \mathrm{mg} \cdot \mathrm{kg}^{-1} \cdot \mathrm{d}^{-1}$ ) for 15 days, the mice in the control group received an equal volume of saline (i.p. daily) for 15 days.

\section{Protocol of LIPUS treatment}

The LIPUS device (Chongqing Haifu Medical Technology Co. LTD, Chongqing, China) was used in this study. The acoustic intensity was $200 \mathrm{~mW} / \mathrm{cm}^{2}$ measured by a sound power meter (UPM-DT-1AV, Ohmic Instruments Co., St. Charles, MO, USA) in degassed water. The frequency of the transducer was $0.3 \mathrm{MHz}$ and the duty cycle was $20 \%$. Before LIPUS treatment, the skin of the lower abdomen and the right ovary was depilated, disinfected, and covered with the ultrasonic coupling agent (Tianjin Chengxin Medical Auxiliary Material Factory, Tianjin, China). Then, LIPUS started to work. The mice of the LIPUS group received ultrasound treatment for 20 minutes per day for 15 consecutive days after successful POF mice modeling. The control group and the POF group were handled as the same as the LIPUS group except for no energy output from LIPUS device.

\section{Estrus cycle examination}

The mice were examined with a vaginal smear of exfoliated cells by fine cotton swabs during a 15-day period following VCD injection. After natural drying, the slides were fixed in $95 \%$ alcohol solution (Chongqing Chuandong Chemical Co. LTD, Chongqing, China) for 10 minutes and stained in $0.23 \%$ alkaline methylene blue (Beijing Solarbio Technology Co. LTD, Beijing, China) for 5 minutes. Vaginal smear was performed under an optical microscope (BX51, Olympus, Tokyo, Japan). The type of estrus cycle was determined as shown by the proportions of nucleated and keratinized epithelial cells and leukocytes. Estrus cycle disorder is a distinguishing characteristic of ovarian function failure [26].

\section{Hematoxylin and Eosin (H\&E) staining}

After 15 days of LIPUS treatment, the mice were over-anesthetized to be euthanized and their ovaries were taken out completely. Sections were observed by H\&E staining to analyze the ovarian morphology and follicle counts. The ovaries tissue was collected from each group and fixed with $4 \%$ paraformaldehyde (Biosharp Co. LTD, Anhui, China) for $48 \mathrm{~h}$. After H\&E staining, sections were observed under an optical microscope. Follicles were counted and classified as primordial, primary, secondary, 
preovulatory, and atretic follicles, respectively ${ }^{[27]}$. Five nonrepetitive views on each slide were selected for statistical analysis.

\section{TUNEL Assay}

Ovarian GCs apoptosis was detected by the TUNEL apoptosis assay kit (4A Biotech Co. LTD, Beijing, China) according to the manufacturer's instructions. The fluorescence was observed under a fluorescence microscope (IX73, Olympus, Tokyo, Japan). Ovarian apoptotic cells were stained bright red. Five fields were randomly selected from sections, and the apoptotic index (AI) was calculated by Image $\mathrm{J}$ (National Institutes of Health, USA).

\section{Immunohistochemistry}

The slides were dewaxed and antigen repaired, then incubated with the primary polyclonal rabbit antibodies of Bax and Bcl-2 (Affinity Biosciences LTD, OH. USA). The concentration of Bax and Bcl-2 was 1:150 and antibodies were incubated for 2 hours at $37^{\circ} \mathrm{C}$ in a water bath pot (Wiggens Co. LTD, Germany). Biotinylated secondary antibody anti-rabbit IgG (Fuzhou Maixin Biotechnology Development Co. LTD, Fuzhou, China) was used on the sections for 40-minute incubation at indoor temperature. Then the slides were colored with DAB (Beijing Zhongshan Jinqiao Biotechnology Co. LTD, Beijing, China). Five sections on each slide were selected randomly for examination under an optical microscope and the Image $\mathrm{J}$ was used for quantitative analysis.

A schematic diagram of research plan could be seen in Figure.1.

\section{Statistical analysis}

All the data was expressed as mean \pm standard deviation. Data were statistically analyzed using the SPSS 22.0 software (IBM, Armonk, New York, USA). Independent-samples t-test was used to analyze the difference in groups. A $p$-value $<0.05$ was considered statistically significant.

\section{Results}

\section{The general situation of mice}

To investigate the general situation of mice, the mice were observed from modeling to treatment. None of the mice had diarrhea during this period. After (i.p.) injection with VCD, the POF mice were depressed, had no glossy hair and poor activity. Compared with the POF group, the mice in the LIPUS group showed glossy hair and increased activity. In the control group, the mice had glossy hair, good activity, a normal diet, and a good response to external stimuli.

\section{Effects of VCD on estrus cycles}

In order to better verify the establishment of POF mice model, the estrus cycles were examined during a 15-day period following VCD injection. The type of estrus cycle was determined as shown by the proportions of nucleated and keratinized epithelial cells and leukocytes (Figure. 2). Regular estrus cycles 
in the control group were shown in all mice and lasted 4-5 days, which includes proestrus for 1 day, estrus for 1-2 days, metestrus for 1 day and diestrus for 1-2 days. However, the significantly irregular estrus cycles were observed after injection with VCD as shown in Figure. 3a. There were irregular estrus cycles with a prolonged diestrus and normal or prolonged estrus in 3 mice, and the other 5 had no cyclicity (Figure. 3b). The results indicated that the VCD-induced POF mice model was established successfully.

\section{Histological changes and follicle count}

Histologic changes and follicular count reflect the reserve capacity of the ovary. The ovary in the control group was of normal size, with multiple growth follicles at different stages in the cortex, and the follicle fluid was deeply stained. In the POF group, the ovarian atrophied formlessly and the number of follicles decreased significantly of which most were atresia follicles with less follicular fluid. Compared with the POF group, the ovarian volume of the LIPUS group was larger, and follicles of various stages were visible (Figure. 4a- $\mathrm{f}$ ). As shown in Figure. 4g, compared with the control group, the number of primordial and primary follicles in the POF group was significantly reduced, the number of atresia follicles was significantly increased $(p<0.05)$, and the number of follicles in other stages decreased $(p<0.05)$. Compared with the POF group, the number of atresia follicles in the LIPUS group was significantly reduced $(p<0.05)$, while the number of primordial follicles, primary follicles and secondary follicles was significantly increased $(p<0.05)$. It was found that VCD could kill small follicles including primordial and primary follicles while LIPUS could help improve ovarian reserve capacity.

\section{Granulosa cell apoptosis}

Apoptosis of GCs is closely related to the development of follicular atresia. The results revealed that the number of apoptotic cells in the POF group was significantly increased compared to the control group (Figure. 5). However, after LIPUS treatment, the apoptosis numbers of GCs were significantly decreased. As shown in the Figure.6, the data obtained by Image $\mathrm{J}$ analysis showed that the AI of LIPUS group was lower than that in the POF group $(p<0.05)$. The results showed that LIPUS prohibited the GCs from apoptosis in POF mice.

\section{Expression of $\mathrm{Bax}$ and $\mathrm{Bcl}-2$}

To further study the mechanism of GCs apoptosis and the follicular atresia, protein expression of Bax and $\mathrm{Bcl}-2$ was detected by immunohistochemical analysis. As shown in Figure. 7, Bax and Bcl-2 proteins were expressed in the follicles of mice in each group, and the positive region showed brown-yellow reaction particles. The data obtained by Image $\mathrm{J}$ analysis (Table. 1) showed that the expression of Bcl2 was decreased and Bax was increased in ovaries of VCD -induced POF mice compared to the control group $(p<0.05)$. After LIPUS treatment, compared with the POF group, the expression of Bcl-2 increased and Bax decreased $(p<0.05)$. Furthermore, the ratio of Bcl-2 /Bax was significantly increased in the LIPUS group than that in the POF group $(p<0.05)$. This finding indicated that LIPUS could improve the apoptosis of GCs by increasing the ratio of Bcl-2 /Bax. 


\section{Discussion}

Approximately $10 \%$ of worldwide women of age between 30 and 39 are suffering from POF ${ }^{[28]}$. A variety of factors have been found to be associated with POF including genetic defects, infections, autoimmunity, drugs, and toxics ${ }^{[29]}$. In our study, the POF mice model was established by VCD. VCD is an ototoxic catabolite of the occupational chemical 4-vinylcyclohexene, an intermediate used in the manufacture of flame retardants, flavors, pesticides, adhesives, fragrances, and synthetic rubber [30]-[31] VCD has long been considered a hazardous occupational chemical that promotes POF and it can well simulate the dynamic process of human POF ${ }^{[11]}$. Prior studies have consistently indicated that VCD induces atretic degeneration of primordial and primary follicles in rodents and primates ${ }^{[13],[32]}$. The results of our study also demonstrated that compared with the control group, the ovarian volume shrank, the primordial and primary follicles decreased $(p<0.05)$, and the atretic follicles increased significantly $(p$ $<0.05)$ in the POF group. On the other hand, estrus cycle disorder is a distinguishing characteristic of ovarian function failure, which was consistent with our results. It has been seen that VCD-induced POF model is safe, has a high success rate, and is relatively stable.

The ovary with periodic development is one of the few organs that fully reflect cell apoptosis. The regulation of follicular development was mainly affected by the local cytokines of the ovary, and the

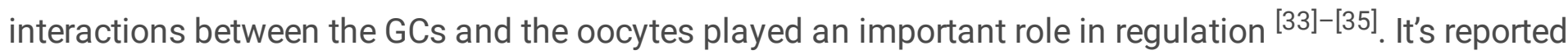
that the follicles become atretic when $10 \%$ of GCs have undergone apoptosis ${ }^{[36]}$. This indicates that GC apoptosis is of great importance in the development of follicular atresia. Regulation of cell apoptosis is a very complex process. Ovarian GC apoptosis follows two main pathways: death receptor and mitochondrial pathways ${ }^{[37]}$. In the earlier pathway, the specific death ligands will combine with death receptors, thus activating caspase cascade ${ }^{[38]}$. In the mitochondrial pathway, it is mainly regulated and activated by the Bcl-2 family, among which pro-apoptotic molecules and anti-apoptotic molecules (Bax and Bcl-2) are the most common apoptotic markers, and Bax will initiate the apoptosis and promote follicular atresia ${ }^{[15],[39]}$. In the process of cell apoptosis, Bax moves to mitochondria from cytoplasm, which subsequently enhances the mitochondrial membrane permeability. BCL-2 prohibits the cytochrome C elimination from entering into the cytoplasm, thus acting antagonistically on BAX and opposing cell apoptosis [40]-[41]. Therefore, Bcl-2/Bax acts as a regulator in follicular maturation and atresia. The ratio of Bcl-2 /Bax determines whether cell apoptosis occurs when stimulated by apoptosis signal, which

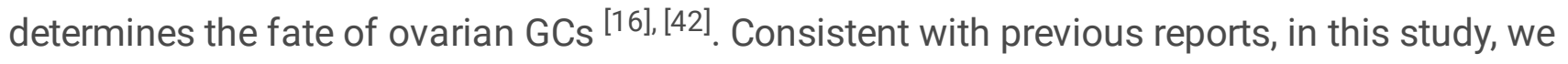
demonstrated that compared with the control group, the mice ovarian GC Al index in the POF group was significantly increased $(p<0.05)$, Bax and Bcl-2 formed homologous dimers, and the ratio of Bcl-2 / Bax decreased $(p<0.05)$, which promoted the apoptosis of ovarian GCs.

In recent years, LIPUS has become a new and non-invasive treatment method. LIPUS is a form of mechanical vibration energy transmission ${ }^{[43]}$. The acoustic pressure wave produced by LIPUS is able to transmit into and through living cells, which may result in a series of biochemical events at the cellular level ${ }^{[43]-[44]}$. In the early 1970s, it was found that small doses of ultrasound boosted the formation of 
follicles in the ovaries of mice ${ }^{[45]}$. The characteristics of ovarian dynamics are related to the repeated proliferation and differentiation of follicular cells ${ }^{[46]}$. Recent study has also shown that LIPUS has a certain effect on the proliferation of mesenchymal stem cells derived from human ovarian follicular fluid [47]. Tang et al. found that LIPUS can promote the recovery of ovarian histological structure in rats with ovarian injury induced by cyclophosphamide ${ }^{[24]}$. In this study, LIPUS helps improve the VCD-induced POF mice ovarian function, reduce ovarian GC apoptosis and restore folliculogenesis. HE staining showed that the primordial and primary follicles of POF mice increased and the atresia follicles decreased after LIPUS treatment. At the same time, apoptosis of granulosa cells plays an important role in the development of ovarian function failure in POF mice. LIPUS could inhibit ovarian GCs apoptosis to recover ovarian function. Furthermore, the ratio of $\mathrm{Bcl}-2$ /Bax was increased by immunohistochemistry. It's suggested that LIPUS can affect mitochondrial pathways regulated by the Bcl-2 family to oppose GC apoptosis.

There are two main limitations to our study. First, it is necessary to further explore whether LIPUS affects another apoptosis pathway of GCs because the two pathways of apoptosis are interrelated. In addition, the increased Bcl-2 /Bax ratio may be related to the promotion of intra-ovarian growth factors by LIPUS, which needs to be confirmed by further studies.

\section{Conclusion}

In conclusion, LIPUS can obviously promote follicular development, inhibit excessive follicular atresia and granular cell apoptosis, and improve the ovarian reserve capacity in POF mice. We found that the expression of anti-apoptotic protein BCL-2 was elevated, and apoptotic protein Bax was decreased, which prohibits the GCs from apoptosis via mitochondrial pathway in the POF mice. It has the potential to provide a promising therapeutic method for patients with POF in the clinic.

\section{Abbreviations}

POF: premature ovarian failure; LIPUS: low intensity pulsed ultrasound; GCs: granulosa cells; VCD: 4vinylcyclohexene diepoxide; Bcl-2: B-cell lymphoma-2; Bax: BCL2-Associated X; HRT: hormone replacement therapy; Al: apoptotic index

\section{Declarations}

\section{Acknowledgements}

None.

\section{Consent for publication}

Not applicable.

\section{Funding}


This work was supported by Science and Technology Project of Guizhou Health and Planning Commission (gzwjkj2020-1-150).

\section{Declaration of competing interest}

We have read and understood Life Sciences' guidelines on the declaration of interests and declare that we have no competing interests.

\section{Data Availability Statement}

All data, models, and code generated or used during the study appear in the submitted article.

\section{Author Contribution}

Y.W. designed the research. H.p.X., C.y.L. and Y.X. performed the experiments. H.p.X., J.X. and Y.X. analyzed data and wrote the paper. Y.W. and J.Q. reviewed the manuscript in preparation.

\section{References}

[1] Laissue P, Vinci G, Veitia RA, et al. Recent advances in the study of genes involved in non-syndromic premature ovarian failure. Mol Cell Endocrinol. 2008;282(1-2):101-111.

[2] Vujović S, Ivović M, Tancić-Gajić M, et al. Premature ovarian failure. Srp Arh Celok Lek. 2012;140(1112):806-811.

[3] Laml T, Schulz-Lobmeyr I, Obruca A, et al. Premature ovarian failure: etiology and prospects. Gynecol Endocrinol. 2000;14(4):292-302.

[4] Kawamura K, Kawamura N, Hsueh AJ. Activation of dormant follicles: a new treatment for premature ovarian failure? Curr Opin Obstet Gynecol. 2016;28(3):217-222.

[5] Lai D, Wang F, Yao X, et al. Human endometrial mesenchymal stem cells restore ovarian function through improving the renewal of germline stem cells in a mouse model of premature ovarian failure. $J$ Transl Med. 2015; 13:155.

[6] Meirow D, Dor J, Kaufman B, et al. Cortical fibrosis and blood-vessels damage in human ovaries exposed to chemotherapy. Potential mechanisms of ovarian injury. Hum Reprod. 2007;22(6):1626-1633.

[7] Yao X, Guo Y, Wang Q, et al. The Paracrine Effect of Transplanted Human Amniotic Epithelial Cells on Ovarian Function Improvement in a Mouse Model of Chemotherapy-Induced Primary Ovarian Insufficiency. Stem Cells Int. 2016; 2016:4148923.

[8] Lee EH, Han SE, Park MJ, et al. Establishment of Effective Mouse Model of Premature Ovarian Failure Considering Treatment Duration of Anticancer Drugs and Natural Recovery Time. J Menopausal Med. 2018;24(3):196-203. 
[9] Torrealday S, Kodaman P, Pal L. Premature Ovarian Insufficiency - an update on recent advances in understanding and management. F1000Res. 2017; 6:2069. Published 2017 Nov 29.

[10] Cordts EB, Christofolini DM, Dos Santos AA, et al. Genetic aspects of premature ovarian failure: a literature review. Arch Gynecol Obstet. 2011;283(3):635-643.

[11] Hoyer PB, Devine PJ, Hu X, et al. Ovarian toxicity of 4-vinylcyclohexene diepoxide: a mechanistic model. Toxicol Pathol. 2001;29(1):91-99.

[12] Cao LB, Leung CK, Law PW, et al. Systemic changes in a mouse model of VCD-induced premature ovarian failure. Life Sci. 2020; 262:118543.

[13] Appt SE, Kaplan JR, Clarkson TB, et al. Destruction of primordial ovarian follicles in adult cynomolgus macaques after exposure to 4-vinylcyclohexene diepoxide: a nonhuman primate model of the menopausal transition. Fertil Steril. 2006;86(4 Suppl):1210-1216.

[14] Hu X, Christian PJ, Thompson KE, et al. Apoptosis induced in rats by 4-vinylcyclohexene diepoxide is associated with activation of the caspase cascades. Biol Reprod. 2001;65(1):87-93.

[15] Cory S, Adams JM. The Bcl2 family: regulators of the cellular life-or-death switch. Nat Rev Cancer. 2002;2(9):647-656.

[16] Tilly JL, Tilly KI, Kenton ML, et al. Expression of members of the bcl-2 gene family in the immature rat ovary: equine chorionic gonadotropin-mediated inhibition of granulosa cell apoptosis is associated with decreased bax and constitutive bcl-2 and bcl-xlong messenger ribonucleic acid levels. Endocrinology. 1995;136(1):232-241.

[17] Sassarini J, Lumsden MA, Critchley HO. Sex hormone replacement in ovarian failure - new treatment concepts. Best Pract Res Clin Endocrinol Metab. 2015;29(1):105-114.

[18] Schmidt KT, Rosendahl M, Ernst E, et al. Autotransplantation of cryopreserved ovarian tissue in 12 women with chemotherapy-induced premature ovarian failure: the Danish experience. Fertil Steril. 2011;95(2):695-701.

[19] Knopman JM, Noyes N, Grifo JA. Cryopreserved oocytes can serve as the treatment for secondary infertility: a novel model for egg donation. Fertil Steril. 2010;93(7): 2413.e7-2413.e2.413E19.

[20] Sheikhansari G, Aghebati-Maleki L, Nouri M, et al. Current approaches for the treatment of premature ovarian failure with stem cell therapy. Biomed Pharmacother. 2018; 102:254-262.

[21] Kovanci E, Schutt AK. Premature ovarian failure: clinical presentation and treatment. Obstet Gynecol Clin North Am. 2015;42(1):153-161. 
[22] Leighton R, Watson JT, Giannoudis P, et al. Healing of fracture nonunions treated with low-intensity pulsed ultrasound (LIPUS): A systematic review and meta-analysis. Injury. 2017;48(7):1339-1347.

[23] Jiang X, Savchenko O, Li Y, et al.: A review of low-intensity pulsed ultrasound for therapeutic applications. IEEE Trans Biomed Eng. 2019 Oct; 66(10):2704-2718.

[24] Tang HJ, Yang H, Fan YJ, et al. Low-intensity pulsed ultrasound promotes repair of cyclophosphamide-induced ovarian injury in rats. Nan Fang Yi Ke Da Xue Xue Bao. 2017;37(12):16321636.

[25] Kappeler CJ, Hoyer PB. 4-vinylcyclohexene diepoxide: a model chemical for ovotoxicity. Syst Biol Reprod Med. 2012;58(1):57-62.

[26] Davis SR. Premature ovarian failure. Maturitas. 1996;23(1):1-8.

[27] Myers M, Britt KL, Wreford NG, et al. Methods for quantifying follicular numbers within the mouse ovary. Reproduction. 2004;127(5):569-580.

[28] Skillern A, Rajkovic A. Recent developments in identifying genetic determinants of premature ovarian failure. Sex Dev. 2008;2(4-5):228-243.

[29] Schover LR. Premature ovarian failure and its consequences: vasomotor symptoms, sexuality, and fertility. J Clin Oncol. 2008;26(5):753-758.

[30] Rappaport SM, Fraser DA. Air sampling and analysis in a rubber vulcanization area. Am Ind Hyg Assoc J. 1977;38(5):205-210.

[31] Devine PJ, Sipes IG, Hoyer PB. Effect of 4-vinylcyclohexene diepoxide dosing in rats on GSH levels in liver and ovaries. Toxicol Sci. 2001;62(2):315-320.

[32] Tran DN, Jung EM, Yoo YM, et al. Depletion of follicles accelerated by combined exposure to phthalates and 4-vinylcyclohexene diepoxide, leading to premature ovarian failure in rats. Reprod Toxicol. 2018; 80:60-67.

[33] Cecconi S, Ciccarelli C, Barberi M, et al. Granulosa cell-oocyte interactions. Eur J Obstet Gynecol Reprod Biol. 2004;115 Suppl 1: S19-S22.

[34] Shaw JL, Dey SK, Critchley HO, et al. Current knowledge of the aetiology of human tubal ectopic pregnancy. Hum Reprod Update. 2010;16(4):432-444.

[35] Inal MM, Incebiyik A, Sanci M, et al. Ovarian cysts in tamoxifen-treated women with breast cancer. Eur J Obstet Gynecol Reprod Biol. 2005;120(1):104-106.

[36] Fu X, He Y, Xie C, et al. Bone marrow mesenchymal stem cell transplantation improves ovarian function and structure in rats with chemotherapy-induced ovarian damage. Cytotherapy. 2008;10(4):353- 
[37] Chen W, Woodruff TK, Mayo KE. Activin A-induced HepG2 liver cell apoptosis: involvement of activin receptors and smad proteins. Endocrinology. 2000;141(3):1263-1272.

[38] Manabe N, Goto Y, Matsuda-Minehata F, et al. Regulation mechanism of selective atresia in porcine follicles: regulation of granulosa cell apoptosis during atresia. J Reprod Dev. 2004;50(5):493-514.

[39] Gillies LA, Kuwana T. Apoptosis regulation at the mitochondrial outer membrane. J Cell Biochem. 2014;115(4):632-640.

[40] Croker BA, O'Donnell JA, Nowell CJ, et al. Fas-mediated neutrophil apoptosis is accelerated by Bid, Bak, and Bax and inhibited by Bcl-2 and Mcl-1. Proc Natl Acad Sci U S A. 2011;108(32):13135-13140.

[41] Xie X, Clausen OP, Boysen M. Prognostic value of Bak expression in oral tongue squamous cell carcinomas. Oncol Rep. 2003;10(2):369-374.

[42] He X, Wang SY, Yin CH, et al. Hydrogen-rich water exerting a protective effect on ovarian reserve function in a mouse model of immune premature ovarian failure induced by Zona Pellucida 3. Chin Med J (Engl). 2016;129(19):2331-2337.

[43] Padilla F, Puts R, Vico L, et al. Stimulation of bone repair with ultrasound: a review of the possible mechanic effects. Ultrasonics. 2014;54(5):1125-1145.

[44] Hu B, Zhang Y, Zhou J, et al. Low-intensity pulsed ultrasound stimulation facilitates osteogenic differentiation of human periodontal ligament cells. PLoS One. 2014;9(4): e95168.

[45] Ter Haar G, Dyson M, Talbert D. Ultrasonically induced contractions in mouse uterine smooth muscle in vivo. Ultrasonics. 1978;16(6):275-276.

[46] Mishra SR, Bharati J, Rajesh G, et al. Fibroblast growth factor 2 (FGF2) and vascular endothelial growth factor A (VEGFA) synergistically promote steroidogenesis and survival of cultured buffalo granulosa cells. Anim Reprod Sci. 2017; 179:88-97.

[47] Omes C, Fassina L, Magenes G, et al. Biological effects of ultrasound stimulus on cells derived from human ovarian follicular liquid. Annu Int Conf IEEE Eng Med Biol Soc. 2013; 2013:850-853.

\section{Tables}

Table.1 Expression of Bax, Bcl-2 and Bcl-2/Bax in three groups ( $x \mathrm{x} \pm \mathrm{s}$ ). 


\begin{tabular}{|lllll|}
\hline Group & $\mathrm{n}$ & $\mathrm{Bax}(\mathrm{AOD})$ & $\mathrm{Bcl}-2(\mathrm{AOD})$ & $\mathrm{Bcl}-2 / \mathrm{Bax}$ \\
\hline Control & 4 & $0.136 \pm 0.014^{\star \star}$ & $0.206 \pm 0.013^{\star \star}$ & $1.520 \pm 0.185^{\star \star}$ \\
\hline POF & 4 & $0.211 \pm 0.017 \boldsymbol{\Delta} \boldsymbol{\Delta}$ & $0.140 \pm 0.013 \boldsymbol{\Delta} \boldsymbol{\Delta}$ & $0.665 \pm 0.051 \boldsymbol{\Delta} \boldsymbol{\Delta}$ \\
\hline LIPUS & 4 & $0.175 \pm 0.013^{*} \boldsymbol{\Delta} \boldsymbol{\Delta}$ & $0.171 \pm 0.009^{\star \star} \boldsymbol{\Delta} \boldsymbol{\Delta}$ & $0.981 \pm 0.089^{\star \star} \boldsymbol{\Delta} \boldsymbol{\Delta}$ \\
\hline
\end{tabular}

Compared with the control group, $\boldsymbol{\Delta} p<0.05, \boldsymbol{\Delta} \boldsymbol{\Delta} p<0.01$, Compared with the POF group $,{ }^{\star} p<0.05, * * p<$ 0.01 .

\section{Figures}

\section{Modeling}

VCD (i.p.) injection

Estrus cycle examination
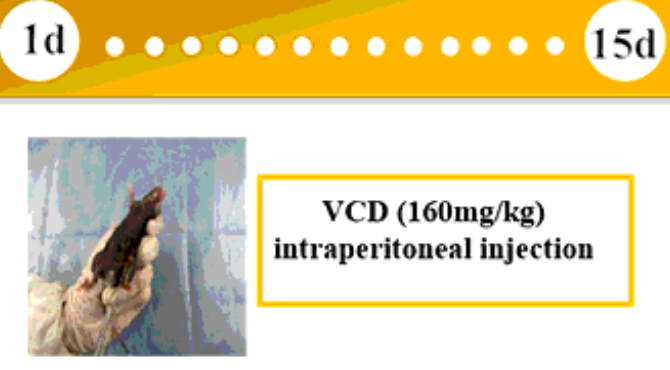

VCD $(160 \mathrm{mg} / \mathrm{kg})$ intraperitoneal injection

C57BL/6 mice

\section{LIPUS treatment}

$\mathrm{H} \& \mathrm{E}$

TUNEL assay

Immunohistochemistry

\section{Figure 1}

A schematic diagram of research plan. 

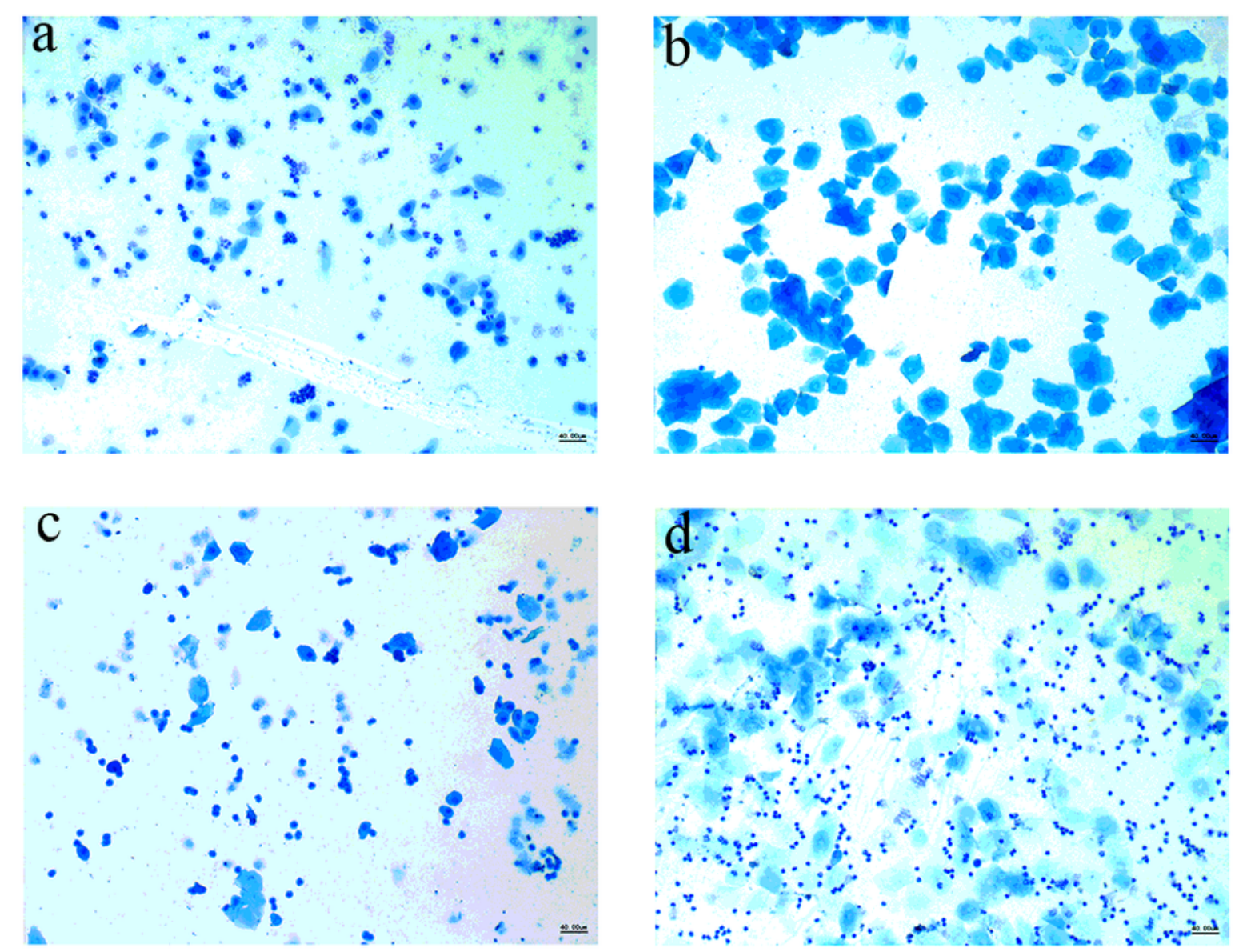

\section{Figure 2}

Effect of 4-vinylcyclohexene diepoxide (VCD) on estrus cycles in mice. Vaginal smears were obtained and the estrus cycles were evaluated by $0.23 \%$ alkaline methylene blue staining. Representative photographs: a proestrus, b estrus, $c$ metestrus, d diestrus (100x). 


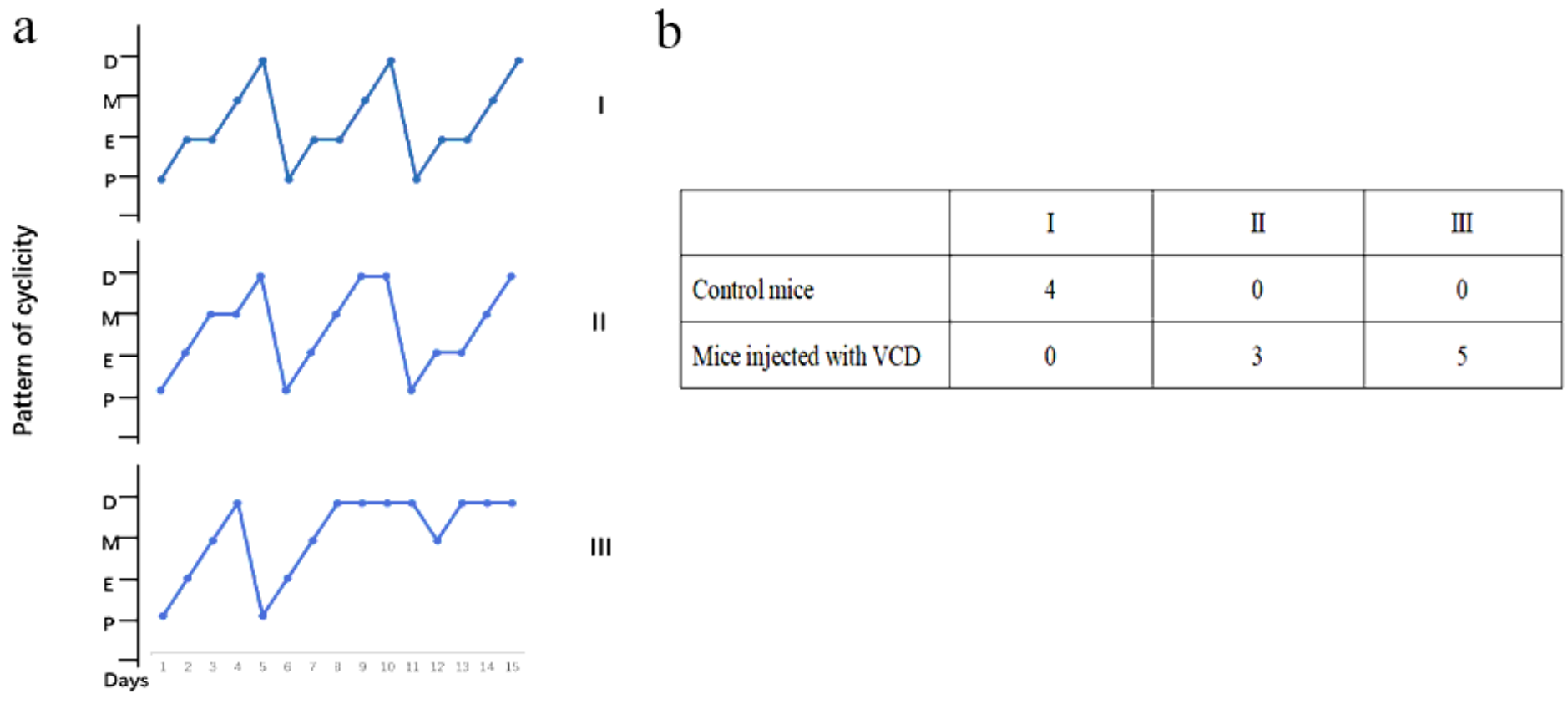

Figure 3

a Three patterns of estrous cycles were graded with the severity of abnormality (I- $\mathbb{Q})$ as follows: $\nabla$, normal

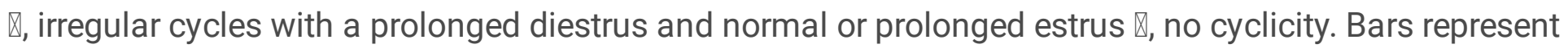
the average length (day) of proestrus $(P)$, estrus $(E)$, metestrus $(M)$ and diestrus $(D)$ per estrous cycle. $b$ Total numbers of mice from each group categorized into the various estrous patterns $(I-\mathbb{Q})$. 


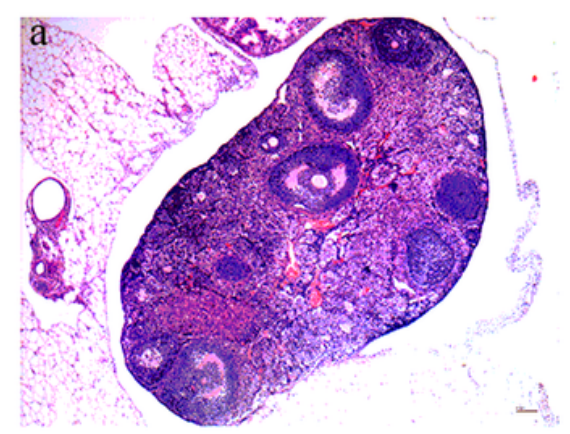

Control

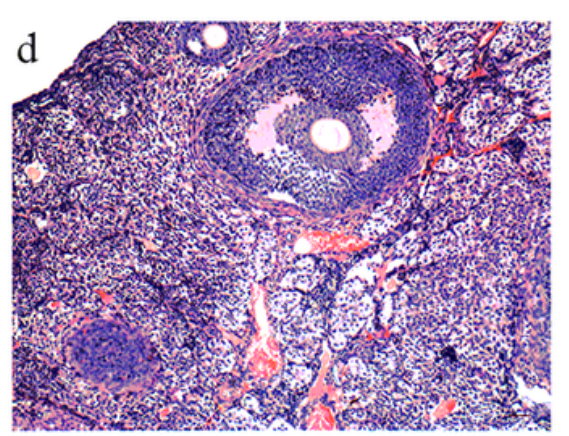

Control

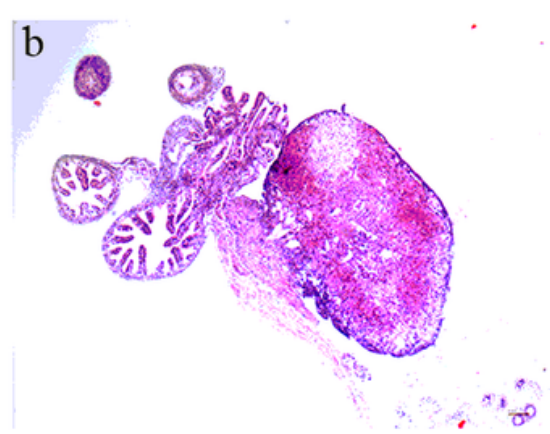

POF

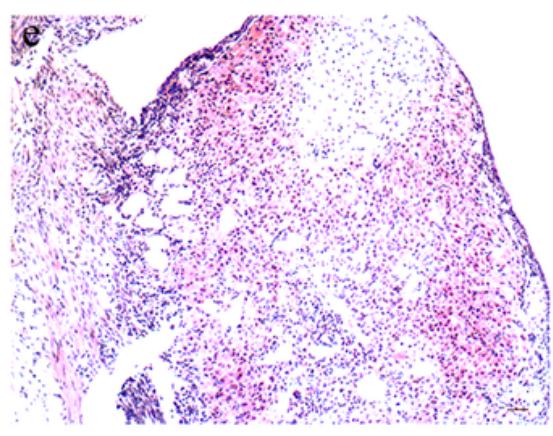

POF

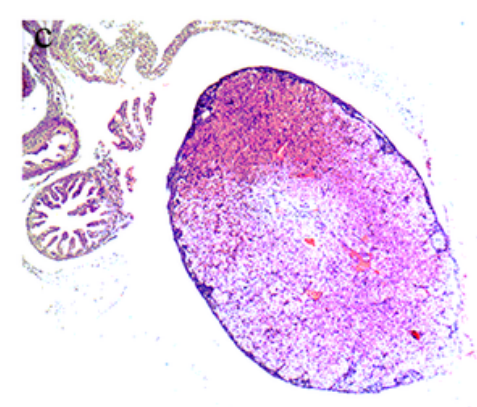

LIPUS

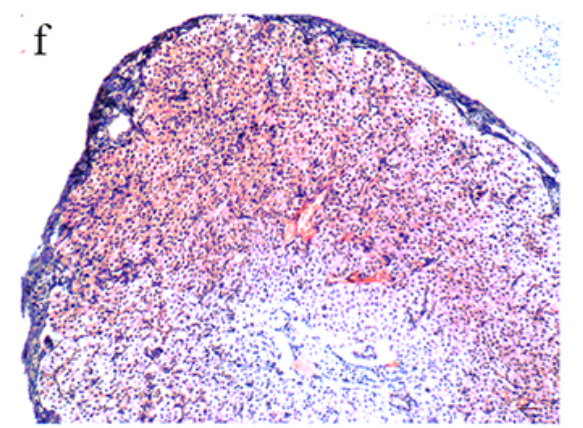

LIPUS

$\mathrm{g}$
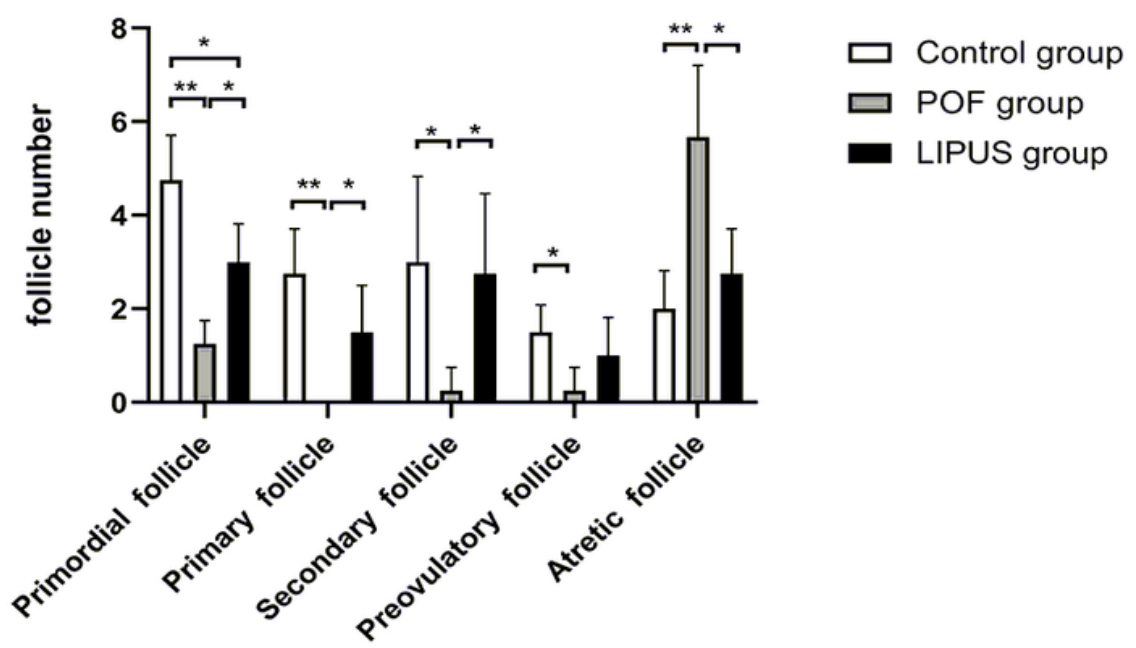

\section{Figure 4}

Effect of low intensity pulsed ultrasound (LIPUS) on follicle growth and ovary in (premature ovarian failure) POF mice. a-f The pathological changes of ovaries (a-c: 40x) and follicles (d-f: 100x) were evaluated by H\&E staining in the three groups. $g$ The number of follicles at various stages were counted and compared in the three groups. ${ }^{*} p<0.05,{ }^{*} p<0.01$. 
DAPI
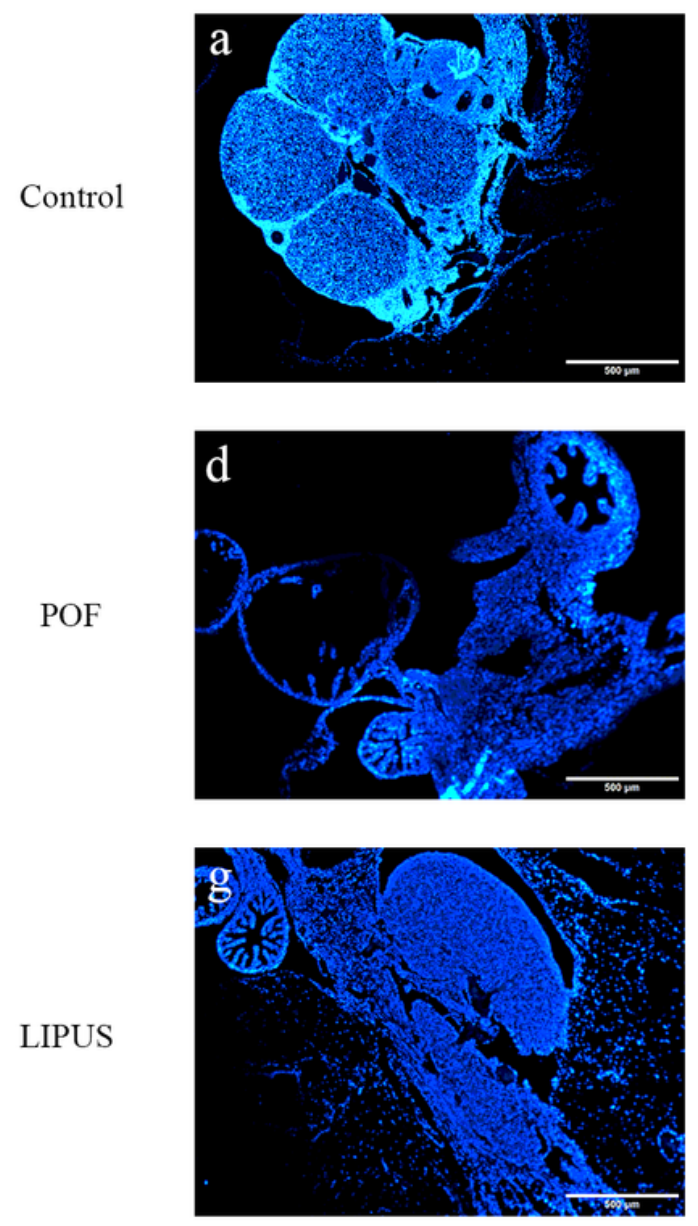

TUNEL
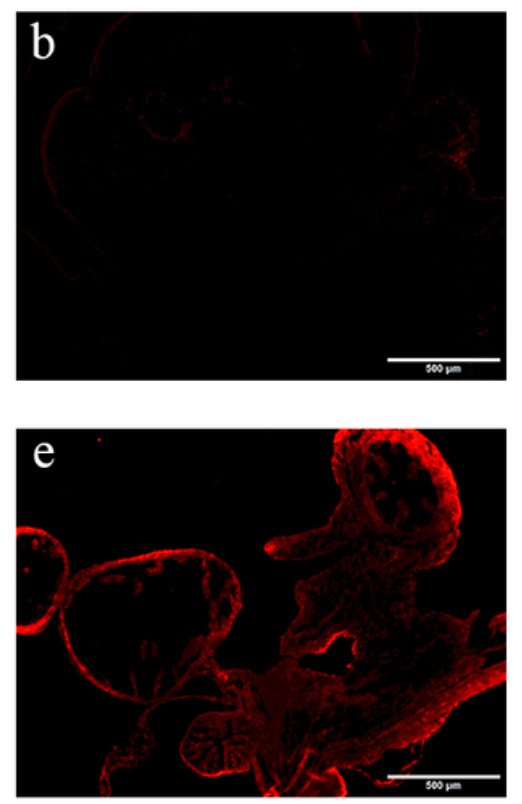

$\mathrm{h}$

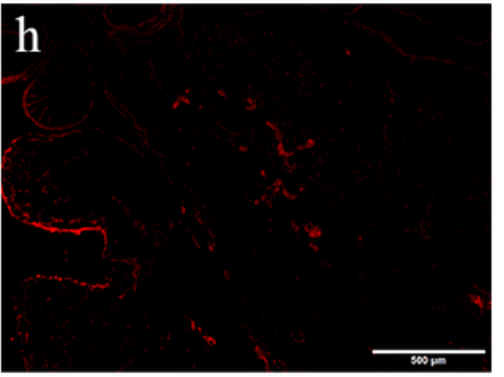

Merge
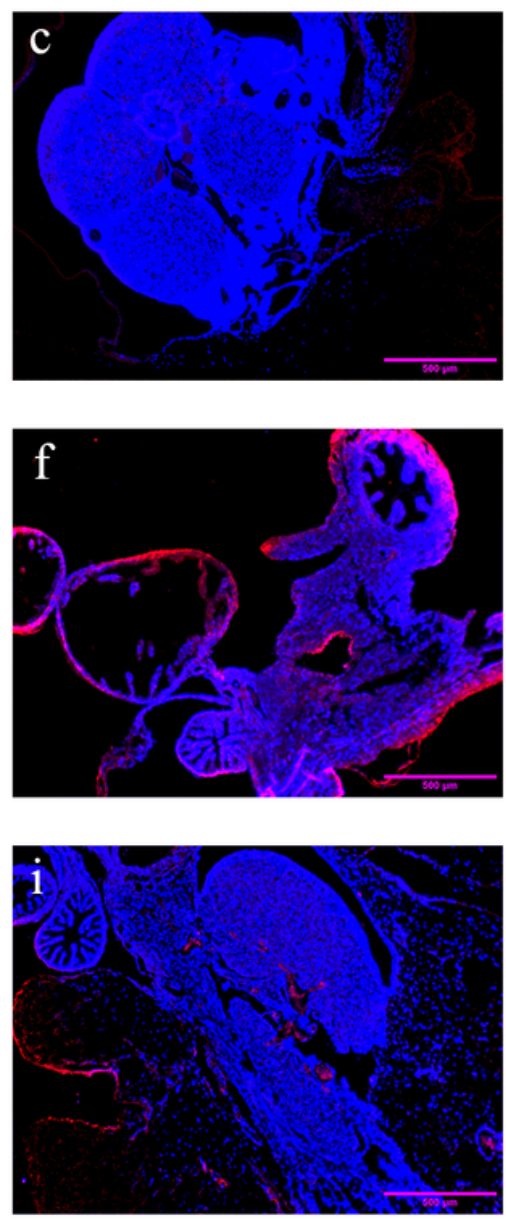

\section{Figure 5}

Apoptosis of granulosa cells (GCs) in ovarian tissues were measured by TUNEL assay. $a, d, g$ The nucleus was stained with DAPI, shown as blue fluorescence (40x). b, e, h Apoptotic cells shown as red fluorescence with TUNEL assay (40x). c, f, i Figures were merged by Image J (40x). 


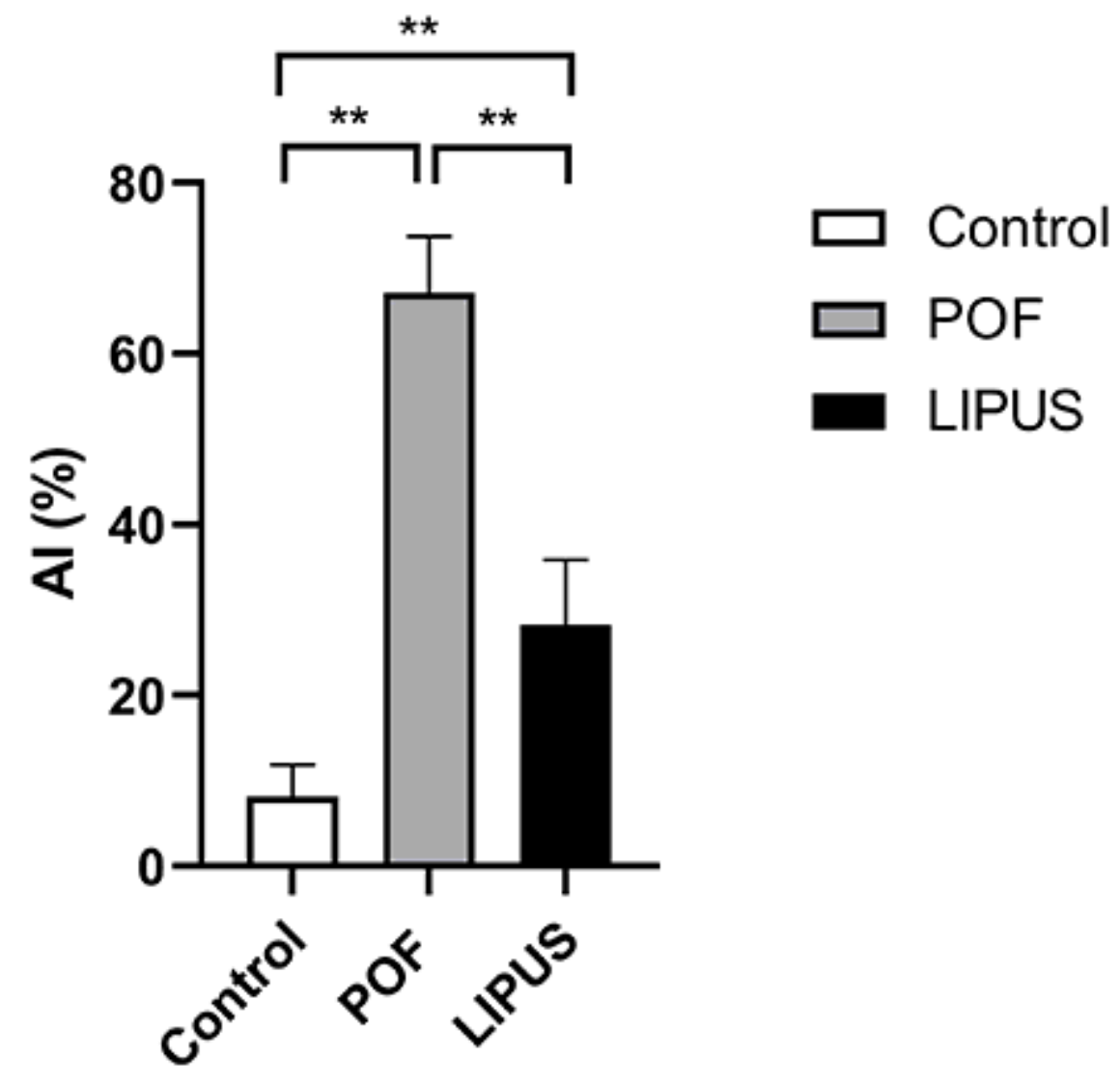

Figure 6

The apoptosis index (Al) of three groups. ${ }^{\star} \mathrm{p}<0.05,{ }^{\star \star} \mathrm{p}<0.01$. 
$\operatorname{Bax}$
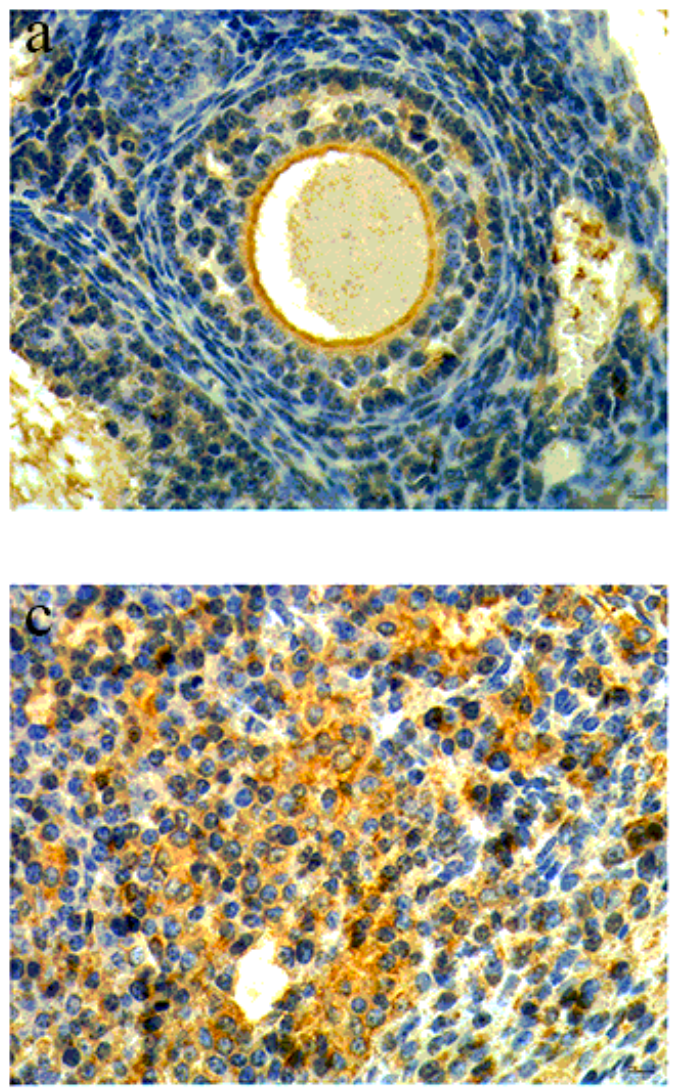

LIPUS

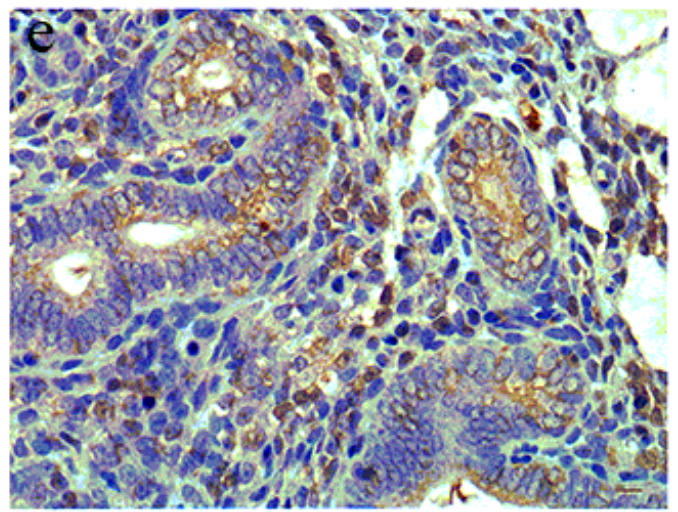

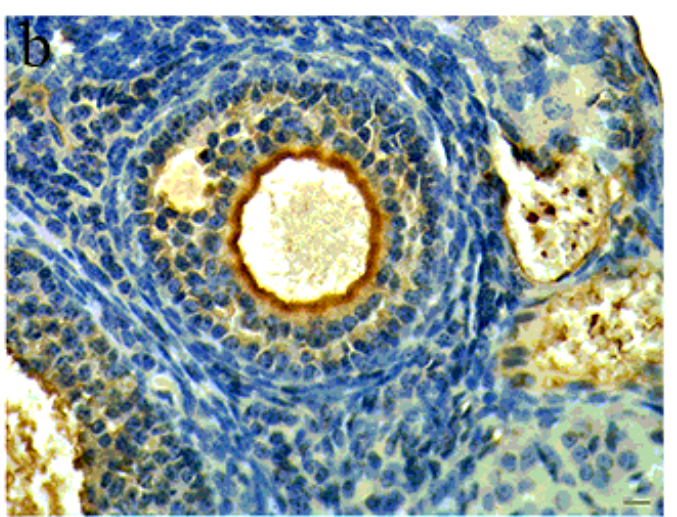
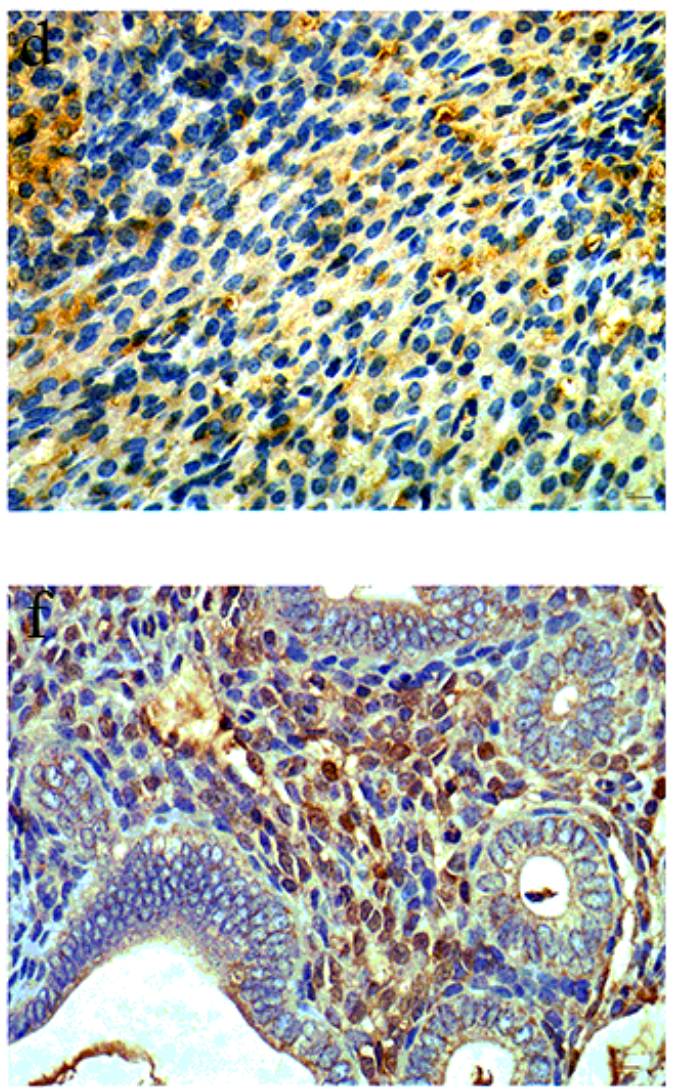

Figure 7

Effect of low intensity pulsed ultrasound (LIPUS) on the protein of Bax and Bcl-2 in premature ovarian failure (POF) mice. Protein expression of Bax and Bcl-2 was evaluated by immunohistochemistry. $a-b$ control group c-d POF group e-f LIPUS group (400x). 